\title{
On the origin of aurorae on Mars
}

\author{
D. A. Brain, ${ }^{1}$ J. S. Halekas, ${ }^{1}$ L. M. Peticolas, ${ }^{1}$ R. P. Lin, ${ }^{1}$ J. G. Luhmann, ${ }^{1}$ D. L. Mitchell, ${ }^{1}$ \\ G. T. Delory, ${ }^{1}$ S. W. Bougher, ${ }^{2}$ M. H. Acuña, ${ }^{3}$ and H. Rème ${ }^{4}$
}

Received 27 September 2005; revised 4 November 2005; accepted 18 November 2005; published 5 January 2006.

[1] We report observations by Mars Global Surveyor (MGS) of thousands of peaked electron energy spectra similar to terrestrial auroral electrons. They are observed on the Martian nightside, near strong crustal magnetic sources. The spectra have peak energies ranging from $100 \mathrm{eV}-$ $2.5 \mathrm{keV}$, and fluxes near the peak are 10-10000 times higher than typical nightside spectra. They occur on magnetic field lines that connect the shocked solar wind to crustal magnetic fields, and on adjacent closed field lines. Their detection is directly controlled by the solar wind, suggesting that magnetic reconnection is required for their observation. We calculate that the most energetic distributions could produce atmospheric emission with intensity comparable to that recently reported from the Mars Express (MEX) spacecraft. Half of the most energetic examples occur during the passage of space weather events past Mars, suggesting that a disturbed plasma environment is favorable for electron acceleration along magnetic field lines. Citation: Brain, D. A., J. S. Halekas, L. M. Peticolas, R. P. Lin, J. G. Luhmann, D. L. Mitchell, G. T. Delory, S. W. Bougher, M. H. Acuña, and H. Rème (2006), On the origin of aurorae on Mars, Geophys. Res. Lett., 33, L01201, doi:10.1029/ 2005 GL024782.

\section{Introduction}

[2] Aurorae are observed throughout the solar system [Clarke et al., 2004], and occur where charged particles have access to an atmosphere along magnetic field lines [McIlwain, 1960]. Though aurorae are most prominent on planets with global dipole magnetic fields (like Earth and Jupiter), localized UV auroral emission was recently reported at Mars [Bertaux et al., 2005], which lacks a global dipole but has strong crustal magnetic fields [Acuña et al., 1998]. The detection of auroral emission by MEX leads naturally to a number of questions. How do the particles and physical mechanisms responsible for Martian aurorae compare to those at other planets? What external conditions and geographic locations are favorable for auroral emission? And is such emission commonplace?

[3] Martian crustal magnetic fields, while much weaker than global dipole fields [Connerney et al., 2001], are sufficiently strong that they stand off the shocked solar wind to $1000 \mathrm{~km}$ altitudes or higher [Brain et al., 2003],

\footnotetext{
${ }^{1}$ Space Sciences Laboratory, University of California, Berkeley, California, USA.

${ }^{2}$ Department of Space Sciences, University of Michigan, Ann Arbor, Michigan, USA.

${ }^{3}$ NASA Goddard Space Flight Center, Greenbelt, Maryland, USA.

${ }^{4}$ Centre D’Etude Spatiale des Rayonnements, Toulouse, France.
}

protecting localized regions of the atmosphere from the impinging solar wind [Mitchell et al., 2001]. In some locations solar wind plasma has direct access to the lower ionosphere along open magnetic field lines (Figure 1). These magnetic cusps are analogous to Earth's polar cusp regions. The emission reported from MEX came from a region of strong radial magnetic field, typically associated with cusps [Krymskii et al., 2002].

[4] The MGS magnetometer and electron reflectometer (MAG/ER) can be used to search for the particles responsible for auroral emission. Since 1999 the MGS mapping orbit has been nearly circular at $400 \mathrm{~km}$ altitudes, and is polar and fixed in local time near $2 \mathrm{am}$ on the nightside. MAG/ER records electron energy distributions from $10 \mathrm{eV}-$ $20 \mathrm{keV}$ every $12-48 \mathrm{~s}$, with energy resolution of $\Delta E / E \sim$ $25 \%$. Angular distributions in 2-D are recorded every $2-8 \mathrm{~s}$, with angular resolution of $14^{\circ} \times 22.5^{\circ}$. The magnetometer records full vector magnetic field every $0.75-3.0 \mathrm{~s}$.

[5] Here, we report auroral-like peaked electron distributions recorded by MAG/ER. We describe a single auroral electron event, including similarities and differences to terrestrial auroral electrons. We then report the locations and conditions for which thousands of peaked spectra are observed by MGS. Finally, we report a correlation between the most energetic spectra and space weather events at Mars.

\section{An Auroral Electron Event}

[6] Figure 2 shows a typical auroral-like electron event recorded by MAG/ER on the Martian nightside, above a strong crustal magnetic source in the southern hemisphere. A population of energetic electrons is evident at 02:24:0002:24:40 and 02:27:30-02:28:30. The peaked distributions are very reminiscent of electron acceleration features observed in Earth's auroral zone [Gurnett and Frank, 1973; Frank and Ackerson, 1971]. The energy of the peak ranges from $100-500 \mathrm{eV}$ for this event, and fluxes at the peak are enhanced by an order of magnitude (or more) relative to nearby spectra.

[7] The topology of the magnetic fields MGS encounters during the event can be identified by examining directional information on electron fluxes relative to the magnetic field (i.e. pitch angle distributions). Cusps are indicated by the presence of loss cone distributions, or an absence of field-aligned electrons returning from the planet indicating that the field line intersects the collisional atmosphere and that those electrons have been scattered or absorbed. Closed field lines are identified by an absence of electrons (plasma voids [Mitchell et al., 2001]) or by two-sided loss cones (trapped distributions). The latter are associated with the outer edges of closed crustal field regions at Mars. In Figure 2, a plasma void is evident from 02:24:50- 


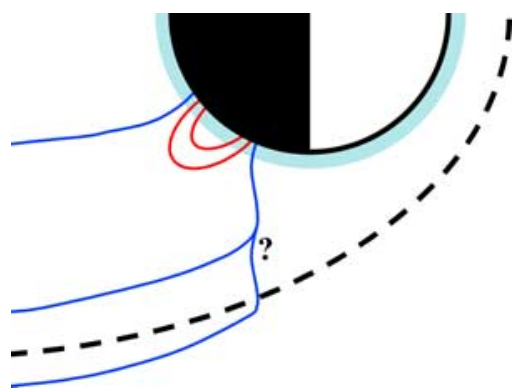

Figure 1. Closed crustal magnetic field lines (red) isolate some portions of the atmosphere from the solar wind. Open field lines (blue) provide access between the atmosphere and solar wind. Nightside cusps may connect to the central tail or to the higher temperature magnetosheath (with inner boundary denoted by the dashed line).

02:27:00, trapped distributions are evident from $2: 24: 40-$ 2:24:50 and 2:27:00-2:27:30, and cusps are evident at most other times. Peaked electron distributions are observed in cusps and on adjacent closed field lines.

[8] Perturbations in the $70 \mathrm{nT}$ magnetic field are evident at the northern and southern edges of the closed field region and co-located with the peaked electron distributions having the highest energy. 10-20 nT westward field deflections can be explained by an upward current sheet with surface current density of $8-16 \mathrm{~mA} / \mathrm{m}$. The magnetic field jump extends over a distance of $5-10 \mathrm{~km}$, constraining the thickness of the current sheet and allowing a calculation of the average volume current. The field perturbations are consistent in amplitude and direction with upward currents of $\sim 1 \mu \mathrm{A} / \mathrm{m}^{2}$ along the separatrix between open and closed field lines. The upward current carried by the electrons at the same location (calculated by integrating over the distribution) is $\sim 0.5 \mu \mathrm{A} / \mathrm{m}^{2}$, roughly consistent with the magnetic field jumps, and of the same order as terrestrial auroral currents [e.g., Potemra, 1985].

[9] Based on the similarities to auroral acceleration features at Earth, and the association with open crustal field lines, the MGS electron observations strongly suggest that auroral-like electron acceleration processes operate at Mars. We note that the downward-going electrons in Figure 2 are nearly isotropic for energies between $100 \mathrm{eV}$ and $1 \mathrm{keV}$. At Earth, auroral electrons are typically field-aligned due to acceleration by parallel electric fields. If the same mechanism is responsible for creating the peaked spectra at Mars, then the angular distribution of electrons is spread between the acceleration region and the location of MGS. This would occur naturally if the electrons have a source in a high temperature, low field (relative to the observation altitude) region. The Martian magnetosheath is such a region, with temperatures and field strengths appropriate to explain the event in Figure 2. Given a sheath temperature of $40 \mathrm{eV}$ and a potential drop of $200 \mathrm{eV}$, we would expect a nearly isotropic down-going electron distribution only if the magnetic field strengths in the sheath were a factor of $\sim 6$ or more lower than the field strengths measured by MGS. Typical sheath field strengths are 10-30 nT, compared to a field magnitude at MGS of roughly $70 \mathrm{nT}$, suggesting that an electron population originating in the sheath may explain the observations. Some additional process, such as waveparticle interactions, may aid in isotropizing the distributions between the acceleration region and the location of MGS, or may be entirely responsible for isotropizing the distributions if they form in a different location.

\section{Conditions and Locations for Aurorae}

[10] We searched the entire MGS dataset for peaked electron energy spectra similar to those shown in Figure 2. In all, we identified nearly 13,000 peaked distributions on the nightside of Mars by searching for spectra with a positive slope in differential flux between $100 \mathrm{eV}$ and $2.5 \mathrm{keV}$. The fluxes at the peaks are as much as three to four orders of magnitude higher than those for typical spectra from the Martian tail or magnetosheath, sometimes saturating the ER instrument. The peaks range in energy from $100 \mathrm{eV}-$ $2.5 \mathrm{keV}$. In general the identified features are isotropic. Auroral-like spectra are observed in MGS data when the
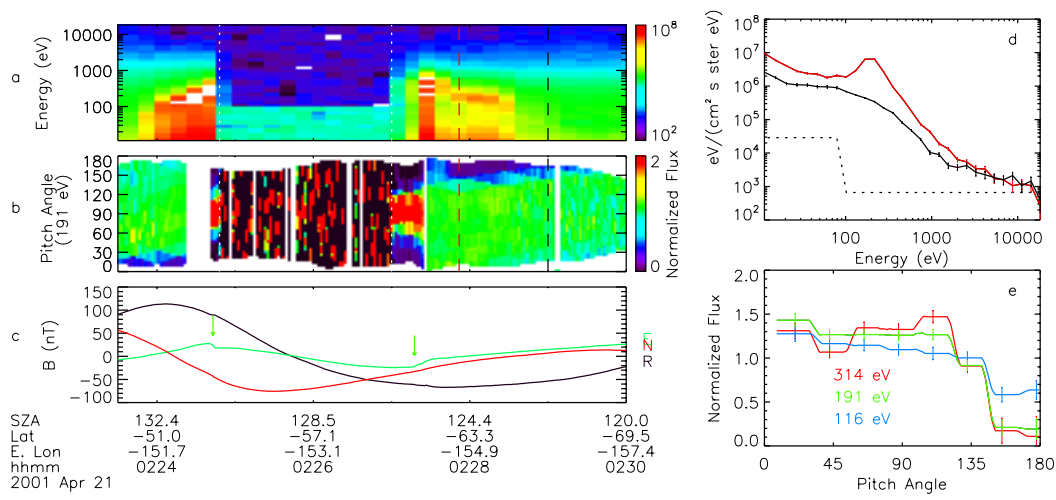

Figure 2. Auroral-like electron event from 21 April, 2001, showing timeseries for: (a) differential energy flux; (b) pitch angle distributions at $191 \mathrm{eV}$; and (c) vector magnetic field with radial (black), eastward (green) and northward (red) components. A red dashed line marks the time of: (d) a peaked electron energy spectrum; and (e) selected pitch angle spectra. A sample unpeaked energy spectrum (black dashed line) is also shown in (d). The dotted line in (d) denotes the instrument background level. Observations indistinguishable from instrument background occurred at times between the white dotted lines in (a). Pitch angle distributions are normalized as a function of time and energy. Arrows mark currentgenerated magnetic field perturbations. 


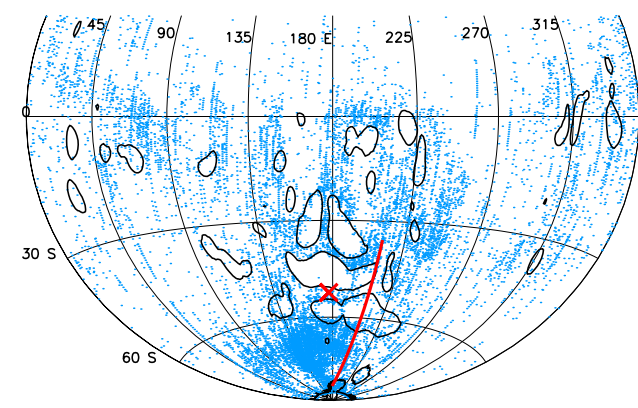

Figure 3. Locations of $\sim 13000$ peaked spectra identified by MGS (blue dots). Contours indicate the typical locations of closed magnetic field lines, inferred from electron angular distributions. The location of the MEX emission (red ' $X$ ') and the orbit track from Figure 2 (red line) are shown for reference.

spacecraft is in darkness, and also when the spacecraft is in sunlight at high solar zenith angles. The emission reported from MEX was observed at a solar zenith angle of $\sim 117^{\circ}$, above the southern nightside.

[11] The geographic distribution of peaked spectra is concentrated near the Martian crustal magnetic fields (Figure 3). Very few were identified in the northern hemisphere, where crustal fields are weak. The features are preferentially observed in regions surrounding areas of typically closed magnetic field, and have been identified both when the spacecraft is on open magnetic field lines and on closed field lines. The presence of auroral electron distributions on closed field lines may indicate that the field lines were recently cusp field lines connected to the IMF and that magnetic reconnection closed the field lines, which contained auroral electrons. A second possibility is that diffusion or some other mechanism transfers electrons onto closed field lines from nearby cusps. A third possibility is that peaked distributions can be created locally on closed field lines.

[12] A number of different external factors influence observations of peaked electron spectra above different locations. We focus on the concentration of peaked spectra evident in Figure 3 above a region centered near $150^{\circ} \mathrm{E}$, $70^{\circ} \mathrm{S}$, which contains a sufficiently high concentration that we can calculate meaningful statistics there. Overall, peaked spectra are identified in $\sim 7 \%$ of observations occurring on cusp (or adjacent closed) field lines. We examine variation with respect to Martian season, IMF draping direction (defined by Brain et al. [2005b]), and upstream pressure proxy (defined by Brain et al. [2005a]). Trends with Martian season (Figure 4a) and IMF direction (Figure 4b) suggest that the orientation of Mars with respect to the IMF strongly affects whether auroral electrons are observed. Terrestrial proton cusp aurorae are directly controlled by the IMF [Fuselier et al., 2002], and are associated with magnetic reconnection of the IMF to Earth's dipole magnetic field. The observations in this location indicate that magnetic reconnection is responsible for providing access of an accelerated electron population to MGS altitudes, and may additionally create the potential structure or waves necessary to accelerate electrons. Peaked spectra are more common during periods of low solar wind pressure (Figure 4c), when crustal sources are less compressed and extend to higher altitudes (closer to possible source regions for auroral electrons) than during other periods.

\section{Discussion}

[13] We used the flux of electrons shown in Figure 2 to calculate its associated auroral emission in the Martian atmosphere. We use a code modified from an Earth electron transport model [Lummerzheim and Lilensten, 1994] that incorporates a model atmosphere at moderate solar activity [Bougher et al., 1990], additional $\mathrm{CO}$ and $\mathrm{CO}_{2}$ cross sections [Sung and Fox, 2000], and a radial non-converging magnetic field. The total energy flux of downward moving electrons at $400 \mathrm{~km}$ was $1.3 \times 10^{-2} \mathrm{ergs} / \mathrm{cm}^{2} / \mathrm{s}$. Roughly half of this energy flux is deposited in the atmosphere, producing $\sim 4 \mathrm{R}$ of column emission of $\mathrm{CO}_{2}^{+} \mathrm{B}^{2} \Sigma_{u}^{+}-\mathrm{X}^{2} \Pi_{g}$ $(289.7 \mathrm{~nm})$ with a peak emission at $145 \mathrm{~km}$. For comparison, the reference spectrum from Figure 2 is an order of magnitude less energetic and produces a factor of 10 less emission. The peaked spectrum in Figure 2 produces atmospheric column emission $\sim 17$ times too weak to produce the intensity of the line-of-sight emission observed by MEX. However, our event was more than 40 times less intense than the most energetic example recorded by MGS. We note that roughly half of the most energetic peaked spectra recorded by MGS occurred during the passage of a solar energetic particle event through the Martian system, as did the emission reported by MEX. This indicates that space weather events may create the necessary conditions for emission like that observed by MEX, either by creating larger potential structures or through promoting reconnection. A similar correlation between auroral intensity and the passage of interplanetary
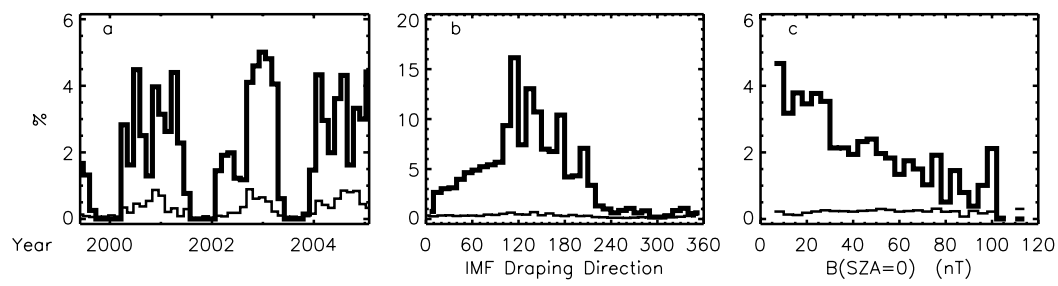

Figure 4. Variability of peaked electron observations with external conditions. The percentage of peaked spectra is shown as a function of all nightside observations occurring at a given (a) time, (b) IMF draping direction, and (c) upstream pressure proxy. The thick line includes variation for the region from $120-180^{\circ} \mathrm{E}$ and $60-90^{\circ} \mathrm{S}$. The thin line includes variation for all other regions. Note the difference in scale for the middle panel. Peaked spectra are preferentially observed during northern summer, in one solar wind sector, and for low solar wind pressure. 
shocks has been reported for the diffuse ultraviolet aurora on Venus [Phillips et al., 1986]. Finally, the downgoing energy flux from auroral electrons contributes to localized atmospheric chemistry and energy deposition, possibly driving regional and temporal variations in upper atmospheric structure and dynamics.

[14] The results presented above are consistent with a source of electrons accelerated by an electric potential along open field lines (possibly associated with magnetic reconnection) into the nightside Martian atmosphere and resulting in optical emission, particularly during times when the near Mars environment is disturbed by passing solar energetic particle events. Peaked electron distributions have also been found in the high altitude central tail by the ASPERA-3 experiment on MEX (R. Lundin, personal communication, 2005), suggesting that this region may be the source of accelerated electrons at Mars. Continued observations by both MGS and MEX will determine the conditions required for the observation of auroral emission, and help to resolve the location and mechanisms responsible for the implied electron acceleration.

[15] Acknowledgments. D.B. and J.H. thank R. Lundin, C. Carlson, T. Phan, M. Fillingim, and R. Lillis for useful discussions. L.P. thanks D. Lummerzheim who provided full access to his transport code. This research was supported through NASA grant NNG04GL35G-05/06.

\section{References}

Acuña, M. H., et al. (1998), Magnetic field and plasma observations at Mars: Initial results of the Mars Global Surveyor mission, Science, 279, 1670-1676.

Bertaux, J.-L., F. Leblanc, O. Witasse, E. Quemerais, J. Lilensten, S. A.

Stern, B. Sandel, and O. Korablev (2005), Discovery of an aurora on Mars, Nature, 435, 790-794, doi:10.1038/nature03603.

Bougher, S. W., R. G. Roble, E. C. Ridley, and R. E. Dickinson (1990), The Mars thermosphere. II-General circulation with coupled dynamics and composition, J. Geophys. Res., 95(14), 14,811-14,827.

Brain, D. A., F. Bagenal, M. H. Acuña, and J. E. P. Connerney (2003), Martian magnetic morphology: Contributions from the solar wind and crust, J. Geophys. Res., 108(A12), 1424, doi:10.1029/ 2002JA009482.

Brain, D. A., J. S. Halekas, R. J. Lillis, D. L. Mitchell, R. P. Lin, and D. H. Crider (2005a), Variability of the altitude of the Martian sheath, Geophys. Res. Lett., 32, L18203, doi:10.1029/2005GL023126.
Brain, D. A., D. L. Mitchell, and J. S. Halekas (2005b), Asymmetry in the draping direction of the IMF at Mars, Icarus, in press.

Clarke, J. T., D. Grodent, S. W. H. Cowley, E. J. Bunce, P. Zarka, J. E. P. Connerney, and T. Satoh (2004), Jupiter's Aurora, pp. 639-670, Cambridge Univ. Press, New York.

Connerney, J. E. P., M. H. Acuña, P. J. Wasilewski, G. Kletetschka, N. F. Ness, H. Rème, R. P. Lin, and D. L. Mitchell (2001), The global magnetic field of Mars and implications for crustal evolution, Geophys. Res. Lett., 28(21), 4015-4018

Frank, L. A., and K. L. Ackerson (1971), Observations of charged particle precipitation into auroral zone, J. Geophys. Res., 76(16), 3612-3643.

Fuselier, S. A., H. U. Frey, K. J. Trattner, S. B. Mende, and J. L. Burch (2002), Cusp aurora dependence on interplanetary magnetic field $\mathrm{Bz}$, J. Geophys. Res., 107(A7), 1111, doi:10.1029/2001JA900165.

Gurnett, D. A., and L. A. Frank (1973), Observed relationships between electric fields and auroral particle precipitation, J. Geophys. Res., 78(1), $145-170$.

Krymskii, A. M., T. K. Breus, N. F. Ness, M. H. Acuña, J. E. P. Connerney, D. H. Crider, D. L. Mitchell, and S. J. Bauer (2002), Structure of the magnetic field fluxes connected with crustal magnetization and topside ionosphere at Mars, J. Geophys. Res., 107(A9), 1245, doi:10.1029/ 2001JA000239.

Lummerzheim, D., and J. Lilensten (1994), Electron transport and energy degradation in the ionosphere: Evaluation of the numerical solution, comparison with laboratory experiments and auroral observations, Ann. Geophys., 12, 1039-1051.

McIlwain, C. E. (1960), Direct measurement of particles producing visible auroras, J. Geophys. Res., 65(14), 2727-2747.

Mitchell, D. L., R. P. Lin, C. Mazelle, H. Rème, P. A. Cloutier, J. E. P. Connerney, M. H. Acuña, and N. F. Ness (2001), Probing Mars' crustal magnetic field and ionosphere with the MGS Electron Reflectometer, J. Geophys. Res., 106(E10), 23,419-23,427.

Phillips, J. L., J. G. Luhmann, and A. I. F. Stewart (1986), The Venus ultraviolet aurora: Observations at $130.4 \mathrm{~nm}$, Geophys. Res. Lett., 13(A4), 1047-1050.

Potemra, T. A. (1985), Field-aligned (Birkeland) currents, Space Sci. Rev, $42,295-311$

Sung, K., and J. L. Fox (2000), Electron impact cross sections for use in modeling the ionospheres/thermospheres of the Earth and planets, Eos Trans. $A G U, 81(48)$, Fall Meet. Suppl., Abstract SA52A-11.

M. H. Acuña, NASA Goddard Space Flight Center, Greenbelt, MD 20771, USA.

S. W. Bougher, Department of Space Sciences, University of Michigan, Ann Arbor, MI 48109-2143, USA.

D. A. Brain, G. T. Delory, J. S. Halekas, R. P. Lin, J. G. Luhmann, D. L. Mitchell, and L. M. Peticolas, Space Sciences Laboratory, University of California, Berkeley, CA 94720, USA. (brain@ssl.berkeley.edu)

H. Rème, Centre D'Etude Spatiale des Rayonnements, F-31028 Toulouse, France. 